\title{
Radiofrequency Ablation Guided by Mechanical Termination of Idiopathic Ventricular Arrhythmias Originating in the Right Ventricular Outflow Tract
}

\author{
MICHAEL KÜHNE, M.D., JEAN-FRANCOIS SARRAZIN, M.D., THOMAS CRAWFORD, M.D., \\ MATTHEW EBINGER, D.O., ERIC GOOD, D.O., AMAN CHUGH, M.D., \\ KRIT JONGNARANGSIN, M.D., FRANK PELOSI JR., M.D., HAKAN ORAL, M.D., \\ FRED MORADY, M.D., and FRANK M. BOGUN, M.D.
}

From the University of Michigan Health System, Ann Arbor, Michigan, USA

\begin{abstract}
Mapping of Idiopathic Ventricular Arrhythmias. Background: Termination of ventricular tachycardia (VT) by mechanical pressure has been described for fascicular and postinfarction VT. Mechanical interruption of idiopathic ventricular arrhythmias (VT/premature ventricular complexes [PVCs]) arising in the right ventricular outflow tract (RVOT) has not been described in systematic fashion.

Methods: Eighteen consecutive patients (13 females, age $49 \pm 13$ years, ejection fraction $0.55 \pm 0.12$ ) underwent mapping and ablation of RVOT VT or PVCs. In 7 patients, 9 distinct VTs (mean cycle length $440 \pm 127 \mathrm{~ms}$ ), and in 11 patients, 11 distinct PVCs originating in the RVOT were targeted. Mechanical termination was considered present if a reproducibly inducible VT was no longer inducible or if frequent PVCs suddenly ceased with the mapping catheter at a particular location. Endocardial activation time, electrogram characteristics, and pace-mapping morphology were assessed at this location. Radiofrequency energy was delivered if mechanical termination was observed.

Results: All targeted arrhythmias were successfully ablated. In 7 of 18 patients (39\%), catheter manipulation terminated the arrhythmia with the mapping catheter located at a particular site. Local endocardial activation time was earlier at sites of mechanical termination $(-31 \pm 7 \mathrm{~ms})$ compared with effective sites without termination $(-25 \pm 3 \mathrm{~ms}, P=0.04)$. The 10 - $\mathrm{ms}$ isochronal area was smaller in patients with mechanical interruption $\left(0.35 \pm 0.2 \mathrm{~cm}^{2}\right)$ than in patients without mechanical termination $\left(1.33 \pm 0.9 \mathrm{~cm}^{2}\right.$, $P=0.01$ ). At all sites susceptible to mechanical trauma, the pace map displayed a match with the targeted VT/PVC. All sites where mechanical termination of VT or PVCs occurred were effective ablation sites.

Conclusions: Mechanical suppression at the site of origin of idiopathic RVOT arrhythmias frequently occurs during the mapping procedure and is a reliable indicator of effective ablation sites. Mechanical termination of RVOT arrhythmias may be indicative of a more localized arrhythmogenic substrate. (J Cardiovasc Electrophysiol, Vol. 21, pp. 42-46, January 2010)
\end{abstract}

catheter ablation, ventricular tachycardia, right ventricular outflow tract, catheter trauma, premature ventricular complexes, mapping

\section{Introduction}

Catheter's contact with the endocardial surface may terminate tachycardias due to mechanical pressure. This phenomenon has been described in atrial tachycardia, atrioventricular nodal re-entrant tachycardia, accessory pathways, and fascicular and postinfarction ventricular tachycardia (VT) and has been used to guide catheter ablation. ${ }^{1-6}$ However, mechanical interruption of VT or premature ventricular complexes (PVCs) originating in the right ventricular outflow tract (RVOT) has not been systematically investigated. The

Dr. Oral reports stock options in Ablation Frontiers. The other authors report no conflicts.

Address for correspondence: Frank Bogun, M.D., Assistant Professor of Medicine, Division of Cardiology, University of Michigan Health System, 3119 TC, 1500 E. Medical Center Dr., Ann Arbor, MI 48109-0366. Fax: 734-936-7026; E-mail: fbogun@med.umich.edu

Manuscript received 5 January 2009; Revised manuscript received 21 May 2009; Accepted for publication 10 June 2009.

doi: 10.1111/j.1540-8167.2009.01566.x purpose of this study was to assess the prevalence of mechanical interruption of idiopathic RVOT arrhythmias and its clinical value for identifying effective target sites for radiofrequency catheter ablation.

\section{Methods}

\section{Patient Characteristics}

The subjects of this study were 18 consecutive patients (13 women, mean age $49 \pm 13$ years $[ \pm 1 \mathrm{SD}]$ ) who were referred for ablation of symptomatic, idiopathic VT or PVCs which originated in the RVOT. Cardiac magnetic resonance imaging was performed in 17 of 18 patients to assess for structural abnormalities. Mean left ventricular ejection fraction was $0.55 \pm 0.12$ and cardiac magnetic resonance imaging did not show any structural abnormalities. The patients underwent unsuccessful treatment with a mean of $1.4 \pm 0.7$ antiarrhythmic medications before the ablation procedure.

A total of 20 arrhythmias were targeted in these patients: 7 of 18 patients had 9 different VT morphologies with a mean cycle length of $440 \pm 127 \mathrm{~ms} ; 11$ patients had 11 distinct PVC 
morphologies occurring in a pattern of bigeminy, trigeminy, or quadrigeminy. All mapped VTs were reproducibly inducible and were sustained when induced. The patients with PVCs had a mean of 24,709 $\pm 24,332$ premature beats ( $20 \pm 14 \%$ of all beats) on 24 -hour Holter monitoring. Isoproterenol was not required to induce VTs or to initiate PVCs. All arrhythmias originated in the RVOT.

\section{Definition}

Catheter-induced mechanical termination was defined as termination of VT or frequent PVCs in the absence of a catheter-induced premature ventricular depolarization (Fig. 1). Furthermore, if VT terminated, it had to be no longer inducible with the catheter at the site of termination.

\section{Electrophysiology Procedure}

The study was approved by the Institutional Review Board of the University of Michigan. After written informed consent was obtained, 2 multipolar catheters were advanced via the femoral veins and positioned at the right ventricular apex and the His-bundle location. Programmed ventricular stimulation from 2 right ventricular sites (right ventricular apex and RVOT) using up to 4 extrastimuli was performed. During the procedure, patients were anticoagulated with intravenous heparin 3,000 units (initial bolus), followed by additional doses of 1,000 units per hour. For mapping and ablation, an open irrigated-tip catheter with a $3.5-\mathrm{mm}$ tip electrode, separated by $1 \mathrm{~mm}$ spacing from a $2-\mathrm{mm}$ ring electrode (Thermocool, Biosense Webster, Diamond Bar, CA, USA) was used in conjunction with a three-dimensional electroanatomic mapping system (CARTO; Biosense Webster). The mapping/ablation catheter was introduced via a long 8.5 French sheath (SR0, St. Jude Medical, DAIG, Minnetonka, $\mathrm{MN}$, USA). The intracardiac electrograms from the mapping catheter and surface electrogram leads were displayed on an oscilloscope and recorded at a speed of $100 \mathrm{~mm} / \mathrm{sec}$. All electrograms were filtered at bandpass settings of 50$500 \mathrm{~Hz}$. The recordings were stored on optical disc (EP Med Inc., West Berlin, NJ, USA). Local endocardial activation time, local electrogram voltage and width, and pace-mapping morphology were assessed at different locations in the RVOT.

\section{Mapping and Ablation}

Activation mapping was performed during VT and PVCs in all patients. If VT terminated or PVCs disappeared during the mapping procedure with the catheter at a certain location, pace mapping was performed and compared to the target arrhythmia at this particular location (Fig. 1). If VT terminated, re-induction was attempted at the right ventricular apex. There were no attempts to exert greater than usual pressure of the ablation catheter against the myocardium.

Bipolar pace mapping was performed at the cycle length of the targeted VT or at the coupling interval of the targeted PVC. A pacing output of up to $10 \mathrm{~mA}$ was used to achieve capture at a pulse width of $2 \mathrm{~ms}$. The morphology of the pace map was compared with the spontaneous PVCs or VT. A matching pace map was considered to be present, if 11 or 12 of 12 leads during pacing were identical to the targeted PVC or VT.

Radiofrequency energy was delivered at the site of earliest endocardial activation or at the site of catheter-induced mechanical termination of VT or PVCs. Applications of radiofrequency energy were delivered at a power of 30-35 watts during ventricular ectopy and/or VT whenever possible. After ablation, programmed right ventricular stimulation was repeated and isoproterenol (2-10 $\mu \mathrm{g} / \mathrm{min})$ was administered in an attempt to reinitiate VT or frequent PVCs. Successful catheter ablation was defined as elimination of ventricular ectopy or VT by radiofrequency ablation, as well as the inability to induce PVCs or VT after the ablation.

\section{Data Analysis}

Activation time, electrogram width, and local voltage during sinus rhythm at the site of origin and the anatomical location of the effective sites (Fig. 2) were assessed. Electronic calipers were used for measurements of electrogram
Figure 1. (A) Left panel: VT termination at an effective ablation site. With the catheter at this site, VT became no longer inducible. (B) Right panel: Pacing at the site shows a matching pace map compared with the induced VT.
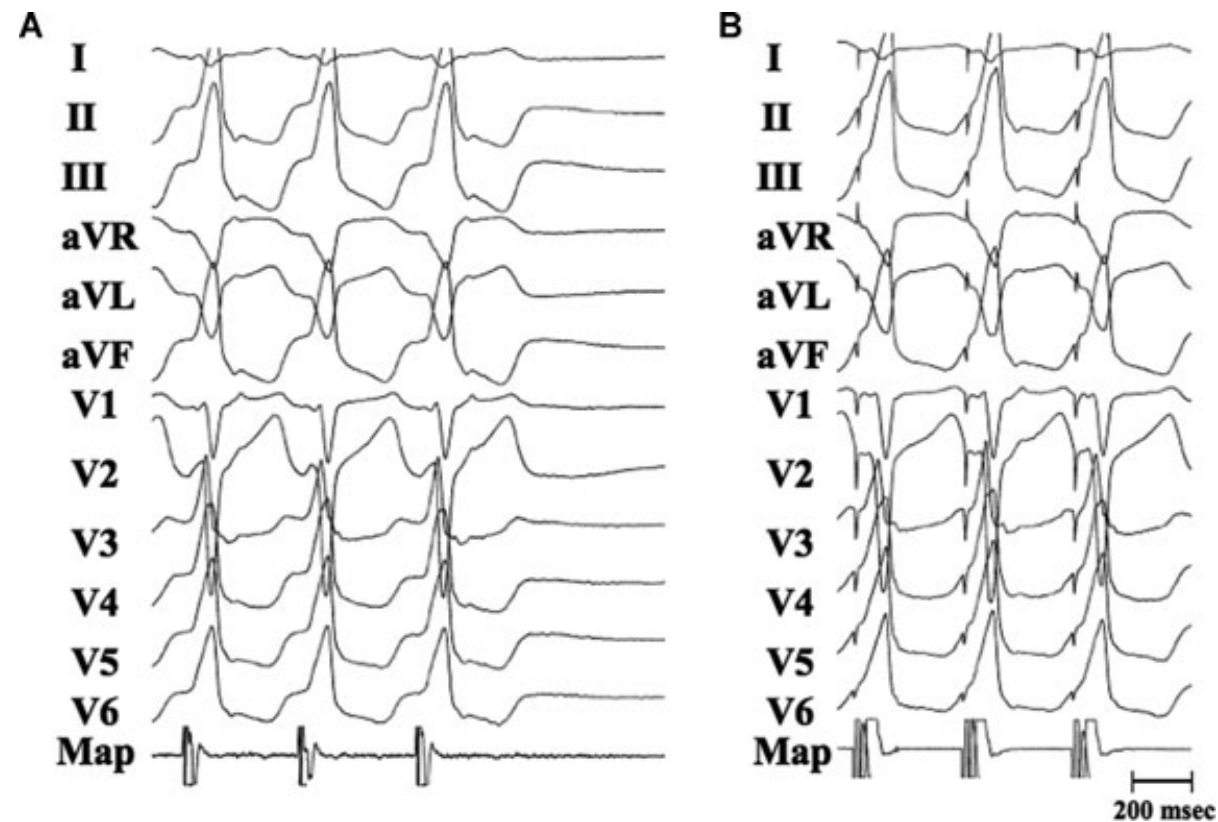


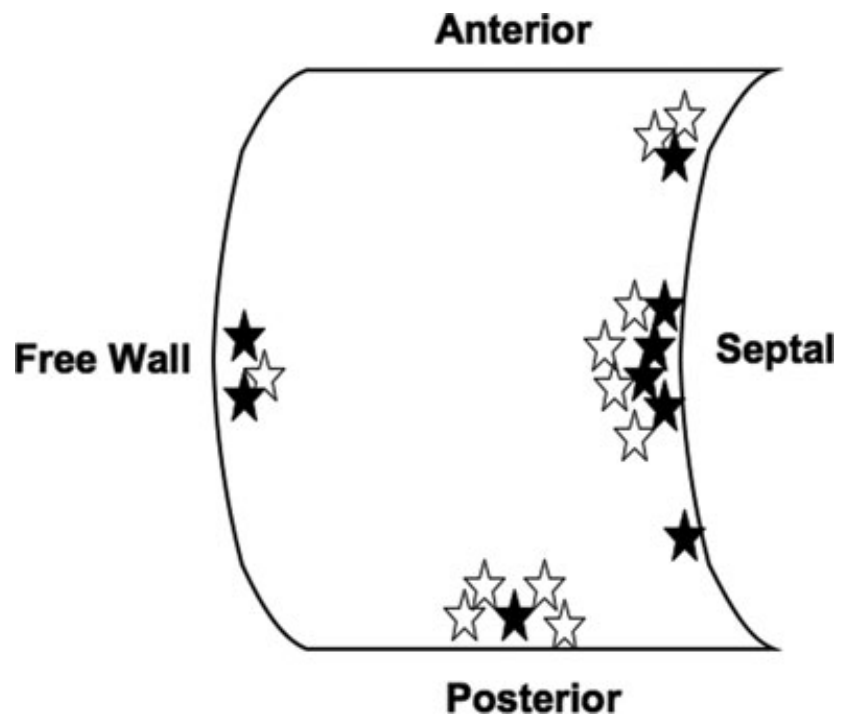

Figure 2. Anatomical location of effective ablation sites where mechanical interruption of VT did occur (black stars) as compared with sites where mechanical interruption did not occur (white stars).

amplitude and width. The area encompassing the first 10-ms isochrone during ventricular arrhythmia was measured on the electroanatomic map (Fig. 3). A mean of $69 \pm 37$ endocardial points were registered during activation mapping $(81 \pm 45$ points in patients with mechanical termination and $61 \pm 26$ points in patients without mechanical termination of ventricular arrhythmias; $\mathrm{P}=0.34$ ). Isolated potentials were sought at the effective ablation sites.

\section{Follow-Up}

All antiarrhythmic drug therapy was discontinued after the ablation procedure.

All patients were seen in an outpatient clinic 3 and 12 months after the procedure and a 24-hour Holter monitor recording was repeated 3 months postprocedure.

\section{Statistical Analysis}

Continuous variables were expressed as mean \pm 1 standard deviation (SD). Continuous variables were compared using Student $t$-test. Discrete variables were compared using the chi-square test or Fisher's exact test, as appropriate. A P-value of $<0.05$ was considered to indicate statistical significance.

\section{Results}

\section{Mapping of VTs and PVCs (Table 1)}

In 7 of 18 patients $(39 \%)$, catheter manipulation resulted in mechanical interruption of PVCs $(n=3)$ or termination of VT $(\mathrm{n}=4)$, rendering VT noninducible or suppressing PVCs with the mapping catheter located at a particular site. Mechanical termination and suppression were observed for 9 arrhythmias in the 7 patients (PVCs at 3 and VT at 6 sites). Hence, mechanical interruption occurred at 9 of $20(45 \%)$ effective ablation sites.

Mechanical termination of arrhythmias occurred at septal $(n=4)$, free wall $(n=2)$, anteroseptal $(n=1)$, posteroseptal $(\mathrm{n}=1)$, and posterior sites $(\mathrm{n}=1)$ in the RVOT (Fig. 2).

The mean local endocardial activation time was $-28 \pm$ $6 \mathrm{~ms}$ at effective sites. Local endocardial activation was significantly earlier at sites where mechanical termination occurred $(-31 \pm 7 \mathrm{~ms})$ than at effective sites without mechanical termination $(-25 \pm 3 \mathrm{~ms}, \mathrm{P}=0.04)$. No isolated potentials were identified during sinus rhythm or during the ventricular arrhythmia at the effective ablation site. There was a matching pace map at all sites of mechanical interruption.

The area of the 10-ms isochrone during VT/PVC was smaller in patients in whom mechanical interruption of arrhythmias occurred than in patients in whom it did not. Local electrogram characteristics did not differ between sites where mechanical interruption of arrhythmia was present compared with sites where mechanical interruption was absent.

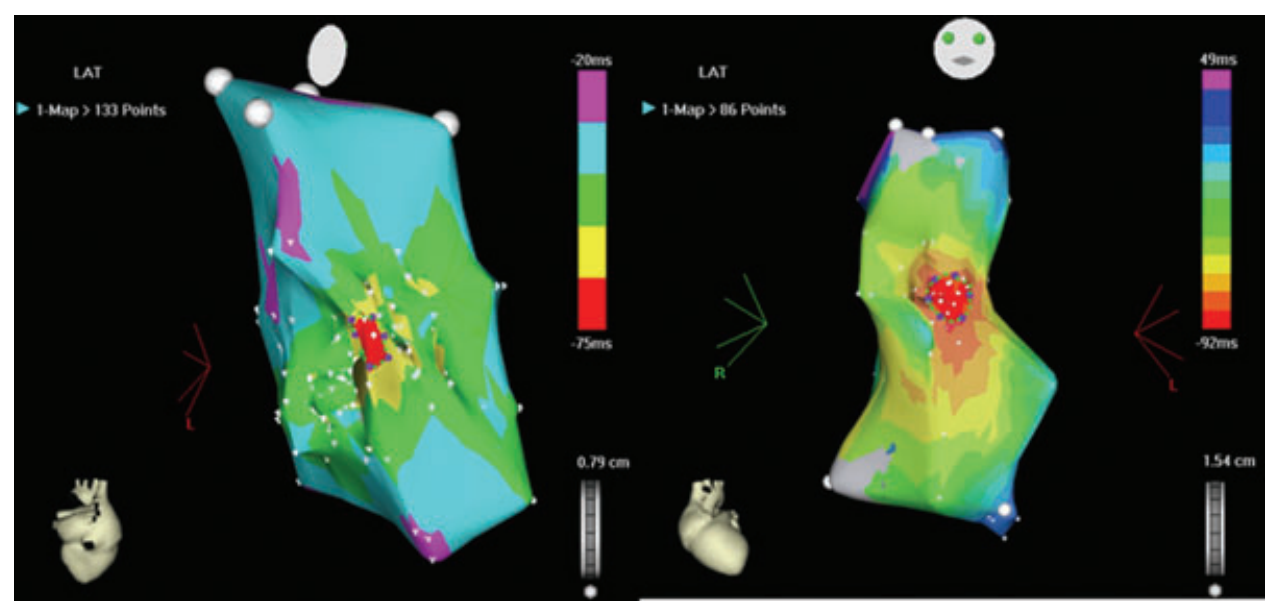

Figure 3. Left panel: Isochronal map in steps of $10 \mathrm{~ms}$ measuring the size of the first 10-ms isochrone $\left(0.4 \mathrm{~cm}^{2}\right)$ in a patient in whom VT could be interrupted by a mechanical contact. The site of origin is the posterior mid RVOT. Right panel: Isochronal map in a patient in whom mechanical termination did not occur. The first 10-ms isochronal area is larger $\left(1.2 \mathrm{~cm}^{2}\right)$ in this patient as compared those in whom mechanical termination did occur (left panel). The effective ablation site was located in the anterior mid RVOT. 
TABLE 1

Electrophysiologic Characteristics of Effective Ablation Sites

\begin{tabular}{lccc}
\hline & \multicolumn{3}{c}{ Mechanical Interruption } \\
\cline { 2 - 4 } & $\begin{array}{c}\text { Present } \\
\text { (9 sites) }\end{array}$ & $\begin{array}{c}\text { Absent } \\
\text { (11 sites) }\end{array}$ & $\begin{array}{c}\text { P- } \\
\text { Value }\end{array}$ \\
\hline Area of 10-ms isochrone $\left(\mathrm{cm}^{2}\right)$ & $0.35 \pm 0.2$ & $1.33 \pm 0.9$ & 0.01 \\
Bipolar voltage in SR (mV) & $2.7 \pm 1.8$ & $2.6 \pm 2.2$ & 0.89 \\
EGM width in SR (ms) & $68 \pm 15$ & $79 \pm 26$ & 0.25 \\
\hline
\end{tabular}

$\mathrm{EGM}=$ electrogram; $\mathrm{SR}=$ sinus rhythm.

\section{Radiofrequency Ablation (Table 2)}

Radiofrequency ablation in the RVOT was effective in all patients. Every site of mechanical interruption of VT or PVCs was an effective ablation site. A mean of $9 \pm 5$ minutes of radiofrequency energy was delivered per patient to eliminate VT or PVCs ( $8 \pm 4$ applications per patient). Fewer ablation lesions were required in patients in whom mechanical arrhythmia interruption occurred, and the procedure time was shorter in these patients. The mean time from the occurrence of mechanical suppression to delivery of radiofrequency energy was $2.9 \pm 3.8$ minutes.

\section{Follow-Up}

During a mean follow-up of $12 \pm 2$ months, 17 of 18 patients $(94 \%)$ remained free of symptomatic arrhythmias. All seven patients (100\%) in whom mechanical termination of VT or suppression of PVCs was observed remained arrhythmia-free during the follow-up. Only one patient with frequent PVCs had recurrent PVCs; his daily PVC burden decreased from $20 \%$ to $6 \%$. The PVCs in this patient had not been terminated by catheter manipulation.

The mean PVC burden decreased from $20 \pm 14 \%$ to $0.8 \pm 2.1 \%(\mathrm{P}<0.01)$. If the patient with recurrent PVCs is excluded, the PVC burden during the follow-up was $0.03 \pm$ $0.03 \%$.

\section{Discussion}

\section{Main Findings}

In this series, approximately $40 \%$ of idiopathic ventricular arrhythmias originating in the RVOT were terminated by mechanical pressure. Termination by mechanical pressure identified sites that were effective ablation sites.

\section{TABLE 2}

Procedural Implications of Mechanical Termination of Ventricular Arrhythmias

\begin{tabular}{|c|c|c|c|}
\hline & \multicolumn{2}{|c|}{ Mechanical Termination } & \multirow[b]{2}{*}{$\begin{array}{c}\text { P- } \\
\text { Value }\end{array}$} \\
\hline & $\begin{array}{c}\text { Present } \\
(\text { Group A) } \\
(\mathbf{n}=7)\end{array}$ & $\begin{array}{c}\text { Absent } \\
(\text { Group B) } \\
(\mathbf{n}=\mathbf{1 1})\end{array}$ & \\
\hline RF time (minutes) & $5.1 \pm 2.2$ & $11.1 \pm 4.9$ & 0.003 \\
\hline RF applications (n) & $4 \pm 2$ & $10 \pm 4$ & 0.0009 \\
\hline Procedure time (minutes) & $155 \pm 48$ & $251 \pm 104$ & 0.02 \\
\hline Fluoroscopy time (minutes) & $27 \pm 15$ & $39 \pm 19$ & 0.16 \\
\hline
\end{tabular}

\section{Mechanical Interruption of Idiopathic Ventricular Arrhythmias}

Myocardial stretch caused by direct distension of myocardium or by volume or pressure overload has been shown to cause electrophysiological changes in myocardial cells in animal models. This phenomenon, termed mechanoelectrical feedback, might be the underlying mechanism of pressure-induced mechanical interruption of PVCs or VT. ${ }^{7}$ RVOT tachycardia has been shown to be caused by triggered activity, mediated by delayed afterdepolarizations. ${ }^{8} \mathrm{~A}$ focus with a subendocardial location would be particularly vulnerable to mechanical trauma. Also, a small discrete focus might be more susceptible than a diffuse site of origin to mechanical impact.

If a critical number of myocardial cells are required for mechanical interruption of impulse generation to occur, our findings indicate that a smaller arrhythmogenic substrate may be present in contact-sensitive ventricular arrhythmias. Accordingly, the 10 -ms isochrone was smaller in patients in whom mechanical termination of ventricular arrhythmias was observed than in those in whom it was not observed. Despite an equal sampling density of sites during activation mapping, the effective ablation sites where mechanical interruption was present showed an earlier activation time compared with sites without mechanical interruption. Earlier activation at the site of origin also suggests that the catheter was closer to the arrhythmia focus. The smaller isochronal area suggests a more discrete substrate, although a lack of sideto-side connections resulting in slowing of propagation of conduction could also possibly result in a smaller isochronal area. The latter is less likely since decreased connectivity is usually associated with some structural abnormality that can be detected by imaging or mapping. Yet sites susceptible to mechanical contact had normal voltage during sinus rhythm and there were no abnormalities on magnetic resonance imaging.

It is possible that the ventricular arrhythmias that were not terminated by mechanical contact arose from sites of origin that either were deeper or larger than when mechanical termination was observed.

\section{Mapping and Ablation}

All sites where mechanical interruption occurred were effective target sites for ablation. In order to minimize unnecessary ablation lesions, we always performed pacing prior to radiofrequency energy delivery to confirm an excellent pace map and catheter stability.

The vulnerability of the site of origin of RVOT arrhythmias to mechanical pressure is clinically helpful, since other mapping criteria may be less accurate and specific. For example, an early activation time does not always result in effective ablation and the first 10-ms isochrone often covers a larger area than the size of an ablation lesion. ${ }^{9}$ Likewise, a matching pace map can be found over a fairly large area. ${ }^{9}$ This new mapping criterion for RVOT arrhythmias occurred in $45 \%$ of the targeted arrhythmias and is therefore clinically useful. The shorter procedure time and the need for fewer ablation lesions make this mapping technique appealing. This mapping technique has not received adequate attention and merits further investigation.

Of note is that no attempts were made to increase catheter pressure against the ventricular wall to elicit mechanical 
suppression. Mechanical interruption occurred with the usual degree of gentle catheter manipulation, and no complications were observed in this study. However, if greater than usual catheter pressure is used in an attempt to elicit mechanical interruption of an arrhythmia, it is possible that the risk of myocardial perforation would increase.

\section{Limitations}

This is a small series of patients and the results need to be confirmed in a larger patient population. With the mapping system used in this study, we were unable to quantify the amount of pressure necessary to terminate ventricular arrhythmias. Another limitation is that we did not test whether the phenomenon of arrhythmia termination was reproducible.

The amount of radiofrequency ablation was greater in this study than in prior reports. ${ }^{10,11} \mathrm{~A}$ possible explanation may be the use of a cooled-tip catheter with a fixed power setting of 30-35 watts; energy was not titrated to achieve a 10-ohm impedance drop and therefore a lesion size might be actually smaller since parameters for lesion formation (i.e., impedance drop) were not assessed.

\section{Conclusion}

Mechanical suppression of idiopathic arrhythmias originating in the RVOT occurs in approximately $40 \%$ of cases and is a reliable indicator of the site of origin of an arrhythmia. This information can be used to facilitate the mapping and ablation procedure in patients with idiopathic ventricular arrhythmias originating in the RVOT.

\section{References}

1. Bogun F, Good E, Han J, Tamirisa K, Reich S, Elmouchi D, Igic P, Lemola K, Oral H, Chugh A, Pelosi F, Morady F: Mechanical inter- ruption of postinfarction ventricular tachycardia as a guide for catheter ablation. Heart Rhythm 2005;2:687-691.

2. Chiang CE, Chen SA, Wu TJ, Yang CJ, Cheng CC, Wang SP, Chiang BN, Chang MS: Incidence, significance, and pharmacological responses of catheter-induced mechanical trauma in patients receiving radiofrequency ablation for supraventricular tachycardia. Circulation 1994;90:1847-1854.

3. Cappato R, Schluter M, Weiss C, Siebels J, Hebe J, Duckeck W, Mletzko RU, Kuck KH: Catheter-induced mechanical conduction block of right-sided accessory fibers with Mahaim-type preexcitation to guide radiofrequency ablation. Circulation 1994;90:282-290.

4. Pappone C, Stabile G, De Simone A, Senatore G, Turco P, Damiano M, Iorio D, Spampinato N, Chiariello M: Role of catheter-induced mechanical trauma in localization of target sites of radiofrequency ablation in automatic atrial tachycardia. J Am Coll Cardiol 1996;27:10901097.

5. Belhassen B, Viskin S, Fish R, Glick A, Glikson M, Eldar M: Catheterinduced mechanical trauma to accessory pathways during radiofrequency ablation: Incidence, predictors and clinical implications. J Am Coll Cardiol 1999;33:767-774.

6. Tai YT, Lee KL, Lau CP: Catheter induced mechanical stunning of accessory pathway conduction: Useful guide to successful transcatheter ablation of accessory pathways. Pacing Clin Electrophysiol 1994;17:31-36.

7. Franz MR: Mechano-electrical feedback in ventricular myocardium. Cardiovasc Res 1996;32:15-24.

8. Lerman BB, Belardinelli L, West GA, Berne RM, DiMarco JP: Adenosine-sensitive ventricular tachycardia: Evidence suggesting cyclic AMP-mediated triggered activity. Circulation 1986;74:270280.

9. Bogun F, Taj M, Ting M, Kim HM, Reich S, Good E, Jongnarangsin K, Chugh A, Pelosi F, Oral H, Morady F: Spatial resolution of pace mapping of idiopathic ventricular tachycardia/ectopy originating in the right ventricular outflow tract. Heart Rhythm 2008;5:339344 .

10. Klein LS, Shih HT, Hackett FK, Zipes DP, Miles WM. Radiofrequency catheter ablation of ventricular tachycardia in patients without structural heart disease. Circulation 1992;85:1666-1674.

11. Azegami K, Wilber DJ, Arruda M, Lin AC, Denman RA: Spatial resolution of pacemapping and activation mapping in patients with idiopathic right ventricular outflow tract tachycardia. J Cardiovasc Electrophysiol 2005; $16: 823-829$. 